\title{
One Conjecture and Two Observations on de Sitter Space
}

\author{
Qing-Guo Huang ${ }^{1}$, Ke $\mathrm{Ke}^{2}$ and Miao $\mathrm{Li}^{1,2}$ \\ 1 Interdisciplinary Center of Theoretical Studies \\ Academia Sinica, Beijing 100080, China \\ and \\ 2 Institute of Theoretical Physics \\ Academia Sinica, P. O. Box 2735 \\ Beijing 100080 \\ huangqg@itp.ac.cn \\ kek@itp.ac.cn \\ mli@itp.ac.cn
}

\begin{abstract}
We propose that the state represented by the Nariai black hole inside de Sitter space is the ground state of the de Sitter gravity, while the pure de Sitter space is the maximal energy state. With this point of view, we investigate thermodynamics of de Sitter space, we find that if there is a dual field theory, this theory can not be a CFT in a fixed dimension. Near the Nariai limit, we conjecture that the dual theory is effectively an $1+1$ CFT living on the radial segment connecting the cosmic horizon and the black hole horizon. If we go beyond the de Sitter limit, the "imaginary" high temperature phase can be described by a CFT with one dimension lower than the spacetime dimension. Below the de Sitter limit, we are approaching a phase similar to the Hagedorn phase in $2+1$ dimensions, the latter is also a maximal energy phase if we hold the volume fixed.
\end{abstract}

May, 2005 
The quantum theory of gravity in de Sitter space is a longstanding problem in modern physics. It may even be the most important problem for the quantum gravity theorist, if the fate of our universe is indeed a de Sitter space. There are two challenging issues in building a quantum theory of de Sitter gravity. If we are to base the theory on the foundation of quantum field theory or string theory, it appears impossible to define measurable observables in an asymptotically de Sitter space [1]. On the other hand, the holographic principle seems to imply that the number of states in the de Sitter gravity is finite, its logarithmic is given by the Gibbons-Hawking entropy, again, a field theory or string theory has no such features. Currently there are two viewpoints regarding these problems. One viewpoint simply presumes that our universe is not asymptotically de Sitter, therefore there do not exist these problems at all, another view is the opposite, and some extreme form even postulates that a small and positive cosmological constant is closely related to supersymmetry breaking. We shall in this note take the second viewpoint. There exists considerable amount of work based on the second viewpoint, for a partial list, see [2] and [1, 5,9$]$.

An important step in approaching the quantum gravity theory in de Sitter space is to work out thermodynamics of de Sitter space. The difficulty here is that there is only one de Sitter space once the cosmological constant of the theory is chosen, thus the usual procedure of calculating thermodynamical quantities is not applicable. However, a family of solutions labelled by the mass of black hole exists, by varying the mass parameter we can vary the cosmic horizon [3, [1. We shall accept the N-bound here, thus the pure de Sitter state is the maximal entropy state with the maximal temperature. What is implicit in the literature but not explicitly stated is that the Nariai black hole is the lowest energy state, namely the ground state. Indeed for the Nariai black hole, the cosmic horizon and the black hole horizon coincide and the Gibbons-Hawking temperature vanishes. We will regard the entropy of the Nariai black hole as the ground state degeneracy, and consider other states with a larger entropy as excited states, in particular, the pure de Sitter state is the limiting state with the largest entropy. Thus, in our thermodynamic considerations, we will subtract the ground state energy as well as the ground state entropy. Subtracting the ground state entropy is an unusual procedure, by doing this we implicitly assume that the entropy of excited states is additive, one part is from the ground state degeneracy, another is due to excited degrees of freedom. We suggest that the subtracted energy and entropy encode the relevant information about the de Sitter gravity. 
In this short note, we calculate the subtracted energy and entropy in four dimensional spacetime. Taking three limits, we make one conjecture in near the Nariai limit, one observation in near the pure de Sitter space limit and another observation in the imaginary high temperature limit. We also generalize our considerations in four dimensions to the cases in higher and $2+1$ dimensions.

Let us start with the metric of Schwarzschild-de Sitter solution in four dimensional spacetime

$$
d s^{2}=-f(r) d t^{2}+f^{-1}(r) d r^{2}+r^{2} d \Omega_{2}^{2}
$$

with

$$
f(r)=1-\frac{r_{0}}{r}-\frac{r^{2}}{L^{2}}
$$

where $r_{0}=2 G m$ and $G$ is the Newton constant. Here $m$ is an integration constant and $L=\sqrt{3 / \Lambda}$ is the size of the pure de Sitter space with a positive cosmological constant $\Lambda$. When $m>0$, a horizon of black hole appears. Raising the parameter $m$, the size of the black hole horizon will increase, while the size of the cosmic horizon will decrease. When $m=m_{N}=\frac{1}{3 \sqrt{3}} \frac{L}{G}$, or $r_{0}=\frac{2}{3 \sqrt{3}} L$, the black hole horizon will be coincident with the cosmic horizon with size $r_{c}=r_{B H}=r_{N}=L / \sqrt{3}$. This is the Nariai black hole.

The temperature of the cosmic horizon of the solution (1) is given by

$$
T_{c}=\frac{1}{4 \pi r_{c}}\left(3 \frac{r_{c}^{2}}{L^{2}}-1\right)
$$

and the relation between parameter $m$ and the size of the cosmic horizon is

$$
m=\frac{r_{c}}{2 G}\left(1-\frac{r_{c}^{2}}{L^{2}}\right)
$$

The cosmic entropy is determined by the the cosmic horizon area

$$
S_{c}=\frac{\pi r_{c}^{2}}{G}
$$

In [5], Balasubramanian et al. use the surface counter term method to find the energy of four dimensional Schwarzschild-de Sitter spacetime and the result is $E=-m$. This result is in accordance with the first law of thermodynamics for the cosmic horizon which is given by

$$
d(-m)=T_{c} d S_{c}
$$


If we choose a positive temperature, the physical energy in de Sitter space should be $-m$, the same as the result in [5].

Bousso proposed that in any asymptotically de Sitter spacetime, the total observable entropy is bounded by the pure de Sitter entropy [6]. The authors of [5] also put forward a conjecture stating that any asymptotically de Sitter space whose mass exceeds that of the pure de Sitter space contains a cosmological singularity. These authors also proposed that the state corresponding to the Nariai black hole has the lowest energy and minimum entropy. We see from eq.(3) that the temperature of the cosmic horizon is zero in the Nariai limit. Based on this fact, we suggest that the Nariai solution represents the ground state of de Sitter spacetime. A state with an energy greater than the Nariai mass is an excited state in de Sitter space.

In general, a thermal system trends to a state with largest entropy if the energy is fixed, or, trends to a state with lowest energy if the entropy is fixed. For the Schwarzschild-de Sitter system, the reliable function of state is free energy

$$
F=E-T S=-m-T_{c} S_{c}=-\frac{r_{c}}{4 G}\left(1+\frac{r_{c}^{2}}{L^{2}}\right),
$$

here we use eq. (35), (4) and (5). This system trends to be a state with lowest free energy, namely the pure de Sitter state, which is consistent with [7].

We now subtract from the energy of a Schwarzschild-de Sitter spacetime the ground state energy. The deviations of the physical mass and the cosmic entropy are given by

$$
\triangle M=M-M_{N}=m_{N}-m=\frac{1}{3 \sqrt{3}} \frac{L}{G}+\frac{r_{c}}{2 G}\left(\frac{r_{c}^{2}}{L^{2}}-1\right)
$$

and

$$
\triangle S_{c}=S-S_{N}=\frac{\pi}{G}\left(r_{c}^{2}-\frac{L^{2}}{3}\right),
$$

where $S_{N}=\pi r_{N}^{2} / G$ is the entropy of the cosmic horizon of the Nariai black hole and here we use eqs.(团) and (5). We suggest that these subtracted thermodynamical quantities of the cosmic horizon encode relevant information about the dual theory of the de Sitter gravity. The first law of thermodynamics (6) can be re-expressed as

$$
d(\triangle M)=T_{c} d\left(\triangle S_{c}\right)
$$


The size of the cosmic horizon can be regarded as a function of the temperature through eq.(3)

$$
r_{c}=r_{N}\left[\frac{T_{c}}{T_{s}}+\sqrt{\left(\frac{T_{c}}{T_{s}}\right)^{2}+1}\right],
$$

where $T_{s}=1 /\left(2 \pi r_{N}\right)=\sqrt{3} /(2 \pi L)$. Then the subtracted energy and the entropy are related to the temperature via

$$
\triangle M=\frac{2 L}{3 \sqrt{3} G}\left(\frac{T_{c}}{T_{s}}\right)^{2}\left[\frac{T_{c}}{T_{s}}+\frac{\left(\frac{T_{c}}{T_{s}}\right)^{2}+\frac{1}{2}+\sqrt{\left(\frac{T_{c}}{T_{s}}\right)^{2}+1}}{\sqrt{\left(\frac{T_{c}}{T_{s}}\right)^{2}+1}+1}\right]
$$

and

$$
\triangle S_{c}=\frac{2 \pi L^{2}}{3 G} \frac{1}{\sqrt{\left(\frac{T_{s}}{T_{c}}\right)^{2}+1}-1} .
$$

Now, both $\Delta M$ and $\Delta S$ are not simple scaling functions of the temperature. we deduce that the quantum theory dual to gravity in de Sitter space can not be described by a single CFT theory. Of course, there must be states of the same energy other than those represented by Schwarzschild-de Sitter solutions, it appears that our conclusion is too strong. However, we believe that the states of Schwarzschild-de Sitter spacetime for a given energy dominates the number of states of the same energy. Note that in the dS/CFT correspondence conjecture [9], the CFT lives in Euclidean space, our conclusion does not apply to that conjecture. We shall study thermal properties of three limiting cases to make one conjecture and two observations in the following.

- Near the Nariai limit - one conjecture

First, we study the system near the Nariai hole whose temperature is much smaller than $T_{s}$. The temperature of the cosmic horizon is almost the same as that of the black hole horizon, more accurately, the difference between these two temperatures is proportional to $T_{c}^{2}$. It says that this system is almost in thermal equilibrium. One might think that the region between the two horizons will collapse to a sphere in the Nariai limit, this turns out not the case. The proper distance between the cosmic horizon and the black hole horizon is

$$
\ell=\frac{L}{\sqrt{3}} \int_{r_{B H}}^{r_{c}}\left[\left(r_{c}-r\right)\left(r-r_{B H}\right)\right]^{-1 / 2} d r=\pi r_{N},
$$


here we take $r_{B H} \rightarrow r_{c}$ in the last step. The subtracted energy and the entropy are given by

$$
\triangle M=\frac{2 \pi^{2}}{3 \sqrt{3}} \frac{L^{3}}{G} T_{c}^{2}
$$

and

$$
\triangle S_{c}=\frac{4 \pi^{2}}{3 \sqrt{3}} \frac{L^{3}}{G} T_{c}
$$

These relations can be interpreted as arising in a conformal field theory (CFT) in $1+1$ dimensional spacetime. We conjecture that in the near extremal (Nariai) limit, the physics can be described by a $2 \mathrm{~d}$ CFT. Now the question is, where does this CFT live? It is interesting to notice that in the near extremal limit, the the spatial part of the metric reduces to $d s^{2}=d \rho^{2}+r_{N}^{2} d \Omega_{2}^{2}$, where $\rho=2 r_{N} \sin ^{-1}\left(\sqrt{\frac{r-r_{-}}{r_{+}-r_{-}}}\right)$. Not only the topology, but also the geometry factorizes into the form $I \times S^{2}$. It appears that the only natural choice is the segment between the two horizons. There is a universal relation between the energy and the temperature in a $1+1$ dimensional CFT which reads $\triangle M=\frac{\pi c}{6} \ell T^{2}$, where $c$ is the central charge of the CFT and $\pi R=\ell, \ell$ is the proper length of the spatial dimension. Applying this relation to eq. (15), we read off the central charge of this CFT to be $c=\frac{12}{\pi} S_{N}$. To specify the CFT, we also have to impose boundary conditions at the two ends of the segment, but we do not have enough information to fix these conditions. However, the thermal properties will not depend on the choice of boundary conditions. Here we stress that Schwarzschild-de Sitter system is not a conservative system and the energy is not conserved. The dual CFT is living in the segment and the energy can also flow outwards or inwards.

To sum up, our conjecture is that near the Nariai limit, the quantum gravity in $d S$ space is dual to an $1+1$ CFT living on the radial segment connecting the cosmic horizon and the black hole horizon.

- Near the pure de Sitter limit - one observation

According to the N-bound conjecture, the state with the highest entropy of this system is the pure de Sitter case, and the temperature is also the highest. Using eqs.(12) and (13), we find that near the pure de Sitter state the subtracted physical mass and entropy are approximately

$$
\triangle M=\frac{L}{3 \sqrt{3} G}+\frac{L}{2 G} \frac{T_{c}-T_{d s}}{T_{d s}}
$$

and

$$
\triangle S_{c}=\frac{2 \pi L^{2}}{3 G}+\frac{\pi L^{2}}{G} \frac{T_{c}-T_{d s}}{T_{d s}},
$$


where $T_{d s}=1 /(2 \pi L)$ is the temperature of the cosmic horizon in pure de Sitter space.

The pure de Sitter state is a limiting state, which reminds us of the Hagedorn temperature in string theory. In the canonical ensemble approach, the string soup can not be heated above the Hagedorn temperature. Near this temperature, the string system tends to form a single long string with the energy scales as

$$
E \sim \frac{1}{T_{H}-T}
$$

where $T_{H}$ is the Hagedorn temperature. This behavior is different from the de Sitter limit where the energy approaches a constant. However, states in de Sitter space are always confined within the cosmic horizon, thus from whatever point of view, the system is one in a finite volume, while the volume occupied by a Hagedorn string diverges. One can estimate the volume occupied by the string as follows. A long string looks like a random walk, so its size $R$ scales with its actual length as $R \sim \sqrt{L}$, the energy of the string is proportional to the length $L$ and the former scales with the oscillator level $N$ as $\sqrt{N}$, thus the size of the string scales with the oscillator level as $R \sim N^{1 / 4}$. At a temperature below $T_{H}, \sqrt{N} \sim 1 /\left(T_{H}-T\right)$. If the string lives in a $d$ dimensional space, the volume occupied by the string behaves as $V=R^{d} \sim 1 /\left(T_{H}-T\right)^{d / 2}$. We see that when $d=2$, the volume has the same divergence as the total energy. The entropy of the string is proportional to $\sqrt{N}$ thus has the same divergence of the energy. If we hold the volume fixed (thus there is only a segment of string in this volume), both the energy and the entropy are also fixed when $d=2$. The string with a fixed volume behaves just like the de Sitter state where the temperature, the energy and the entropy all have a finite limit. Moreover, we expect that the corrections to the energy and the entropy both tend to zero linearly in $T_{H}-T$.

In conclusion, our first observation is that the de Sitter system near the pure de Sitter phase behaves just like the Hagedorn string in a fixed volume in $2+1$ dimensions.

- Imaginary high temperature limit - another observation

What is interesting about the de Sitter gravity is that thermodynamics can be analytically extended to temperatures higher than the pure de Sitter temperature. A higher temperature is realized by the cosmic temperature of a spacetime when parameter $m$ becomes negative. In this spacetime, $r=0$ is a naked singularity. It is convenient to replace $m$ by $-m$ to make $m$ positive, or equivalently $r_{0} \rightarrow-r_{0}$. Taking a limit with $r_{0} \gg L$, the size of the cosmic horizon is approximately given by

$$
r_{c} \simeq\left(r_{0} L^{2}\right)^{1 / 3}
$$


The temperature of the cosmic horizon becomes

$$
T_{c}=\frac{3}{4 \pi L}\left(\frac{r_{0}}{L}\right)^{1 / 3}
$$

and we have $T_{c} \gg T_{s}$. Eqs.(12) and (13) become

$$
\begin{aligned}
& \triangle M=\frac{32 \pi^{3} L^{4}}{27 G} T_{c}^{3} \\
& \triangle S_{c}=\frac{16 \pi^{3} L^{4}}{9 G} T_{c}^{2} .
\end{aligned}
$$

These thermal relations are just the ones in a $2+1$ dimensional CFT. Of course as we already said that there is a naked singularity at $r=0$ not allowed by the cosmic censorship principle. So we can get our second observation that the imaginary high temperature phase is described by a 2+1 dimensional CFT. When the temperature drops below the de Sitter temperature, comparing (22) with (13), we find that the entropy of this $2+1$ dimensional CFT is always less than that of the $d S_{4}$. This fact tells us that the number of degrees of freedom in this $2+1 \mathrm{CFT}$ is not large enough to describe the quantum theory of gravity in $\mathrm{dS}_{4}$.

Next we will generalize our previous considerations to a higher dimensional de Sitter space. Let the spacetime dimension be $n+1$. The metric of the Schwarzschild-de Sitter solution takes the form

$$
d s^{2}=-f(r) d t^{2}+f^{-1}(r) d r^{2}+r^{2} d \Omega_{n-1}^{2}
$$

where

$$
\begin{gathered}
f(r)=1-\frac{r_{0}^{n-2}}{r^{n-2}}-\frac{r^{2}}{L^{2}} \\
r_{0}^{n-2}=\omega_{n-1} m, \quad \omega_{n-1}=\frac{16 \pi G}{(n-1) \operatorname{Vol}\left(S^{n-1}\right)},
\end{gathered}
$$

$L=\sqrt{\frac{n(n-1)}{2 \Lambda}}$ is the size of the pure $\mathrm{dS}_{n+1}$ and $m$ is an integration constant. When $m>0$, there is a cosmic horizon and a black hole horizon. When $m$ increases, the size of the cosmic horizon decreases, but the size of black hole increases. When $m$ reaches a critical value $m_{N}=\frac{2}{n \omega_{n-1}}\left(\frac{n-2}{n} L^{2}\right)^{\frac{n-2}{2}}$, corresponding to the Nariai solution, the cosmic horizon and the black hole horizon share the same hypersurface with size $r_{c}=r_{B H}=r_{N}=\sqrt{\frac{n-2}{n}} L$. Using 
metric (23), we can express parameter $m$, the temperature and entropy corresponding to the cosmic horizon as

$$
\begin{aligned}
m & =\frac{r_{c}^{n-2}}{\omega_{n-1}}\left(1-\frac{r_{c}^{2}}{L^{2}}\right) \\
T_{c} & =\frac{1}{4 \pi r_{c}}\left[n \frac{r_{c}^{2}}{L^{2}}-(n-2)\right]=\frac{n}{4 \pi L^{2} r_{c}}\left(r_{c}^{2}-r_{N}^{2}\right), \\
S_{c} & =\frac{r_{c}^{n-1} \operatorname{Vol}\left(S^{n-1}\right)}{4 G}=\frac{4 \pi r_{c}^{n-1}}{(n-1) \omega_{n-1}} .
\end{aligned}
$$

The first law of thermodynamics can be expressed as

$$
d(-m)=T_{c} d S_{c}
$$

In [5], the authors calculate the mass of the gravitational field in $(4+1)$ dimensional spacetime and find $M_{5}=\frac{3 \pi L^{2}}{32 G}-m$ by the counter term method. In higher dimensions, more counter terms are needed. Since we only need to know the difference between the energy of a general Schwarzschild-de Sitter spacetime and that of the Nariai hole, we can ignore the constant correction to the mass. From eq.(26), the subtracted mass and the entropy are given by

$$
\begin{aligned}
& \triangle M=\frac{r_{N}^{n-2}}{\omega_{n-1}}\left[\frac{2}{n}+\frac{n-2}{n}\left(\frac{T_{c}}{T_{s}}+\sqrt{\left(\frac{T_{c}}{T_{s}}\right)^{2}+1}\right)^{n}-\left(\frac{T_{c}}{T_{s}}+\sqrt{\left(\frac{T_{c}}{T_{s}}\right)^{2}+1}\right)^{n-2}\right] \\
& \triangle S_{c}=\frac{4 \pi r_{N}^{n-1}}{(n-1) \omega_{n-1}}\left[\left(\frac{T_{c}}{T_{s}}+\sqrt{\left(\frac{T_{c}}{T_{s}}\right)^{2}+1}\right)^{n-1}-1\right],
\end{aligned}
$$

where $T_{s}=\frac{n-2}{2 \pi r_{N}}$.

Taking the near Nariai limit, $T_{c} \ll T_{s}$, the proper distance between the cosmic and black hole horizons approaches $\ell=\pi r_{N} / \sqrt{n-2}$. The subtracted mass and entropy in this limit read

$$
\begin{aligned}
\triangle M & =\frac{n-1}{\sqrt{n-2}} S_{N} \ell T_{c}^{2}, \\
\triangle S_{c} & =\frac{2(n-1)}{\sqrt{n-2}} S_{N} \ell T_{c}
\end{aligned}
$$

where $S_{N}=\frac{4 \pi r_{N}^{n-1}}{(n-1) \omega_{n-1}}$ is the entropy associated with the cosmic horizon in the Nariai solution. These quantities describe a system excited over the Nariai ground state. The metric 
also factorizes $d s^{2}=d \rho^{2}+r_{N}^{2} d \Omega_{n-1}^{2}$, where $\rho=\frac{2 r_{N}}{\sqrt{n-2}} \sin ^{-1}\left(\sqrt{\frac{r-r_{-}}{r_{+}-r_{-}}}\right)$. Again, eq.(29) tells us that the excitation near the Nariai system can be described by a two dimensional CFT with the central charge $c=\frac{6(n-1)}{\pi \sqrt{n-2}} S_{N}$. When $m$ is small and the spacetime is close to the pure de Sitter space, the subtracted mass and the entropy are approximated by

$$
\begin{aligned}
& \triangle M=\frac{r_{N}^{n-2}}{\omega_{n-1}}\left[\frac{2}{n}+\frac{2}{n-1}\left(\frac{n}{n-2}\right)^{\frac{n-2}{2}} \frac{T_{c}-T_{d s}}{T_{d s}}\right], \\
& \triangle S_{c}=\frac{4 \pi r_{N}^{n-1}}{(n-1) \omega_{n-1}}\left[\left(\frac{n}{n-2}\right)^{\frac{n-1}{2}}-1+\left(\frac{n}{n-2}\right)^{\frac{n-1}{2}} \frac{T_{c}-T_{d s}}{T_{d s}}\right],
\end{aligned}
$$

where $T_{d s}=1 /(2 \pi L)$ is the temperature of the cosmic horizon in pure de Sitter space. The scaling behavior once again is similar to the Hagedorn string in a fixed volume in $2+1$ dimensions.

We also like to examine the unphysical high temperature phase with $r_{0}^{n-2} \rightarrow-r_{0}^{n-2}$ and $r_{0} \gg L$. There is a naked singularity at $r=0$ and the temperature of the cosmic horizon reads $T_{c} \simeq \frac{n}{4 \pi L}\left(\frac{r_{o}}{L}\right)^{\frac{n-2}{n}}$. Now we have

$$
\begin{aligned}
& \triangle M=\frac{1}{n} \frac{(4 \pi)^{n}}{(n-2)^{n-1}} \frac{r_{N}^{2 n-2}}{\omega_{n-1}} T_{c}^{n} \\
& \triangle S_{c}=\frac{1}{n-1} \frac{(4 \pi)^{n}}{(n-2)^{n-1}} \frac{r_{N}^{2 n-2}}{\omega_{n-1}} T_{c}^{n-1} .
\end{aligned}
$$

In this limit, one may postulate that gravity in spacetime is described by a CFT in $(n-1)+1$ dimensional spacetime. Again we find that the entropy in eq.(31) after extended back to the physical region is always smaller than that in eq. 28). This result shows that this $(n-1)+1$ CFT cannot describe gravity of Schwarzschild-de Sitter spacetime in $n+1$ dimensions.

Finally, we study the case of the $2+1$ dimensional de Sitter space (some points appeared previously in [8]). There is no black hole in the $2+1$ dimensional de Sitter space, however, there exist solutions also called Schwarzschild-de Sitter with only one horizon and the metric is given by

$$
d s^{2}=-\left(1-8 G m-\frac{r^{2}}{L^{2}}\right) d t^{2}+\left(1-8 G m-\frac{r^{2}}{L^{2}}\right)^{-1} d r^{2}+r^{2} d \phi^{2}
$$

where $L=1 / \sqrt{\Lambda}$ is the size of the pure de Sitter space with cosmological constant $\Lambda$. There is no black hole horizon in this spacetime. The size of the cosmic horizon is

$$
r_{c}=L \sqrt{1-8 G m}
$$


the temperature of the cosmic horizon is

$$
T_{c}=\frac{r_{c}}{2 \pi L^{2}}=\frac{\sqrt{1-8 G m}}{2 \pi L}
$$

and the entropy corresponding to the cosmic horizon is

$$
S_{c}=\frac{2 \pi r_{c}}{4 G}=\frac{\pi L \sqrt{1-8 G m}}{2 G} .
$$

The first law of thermodynamics takes the form

$$
d(-m)=T_{c} d S_{c}
$$

so $-m$ can be viewed as energy. The spacetime (33) can be thought of as generated by a massive point particle, and there is a deficit angle. When $m=1 /(8 G)$, the deficit angle becomes $2 \pi$ and the space is closed up. For a generic $m$, we are not supposed to take the system to describe the maximal entropy state of a given energy, since there is no hole around. Nevertheless, taking the limit $m=1 /(8 G)$ we find that the temperature of the cosmic horizon vanishes and the entropy tends to zero too, we may postulate that this state is the ground state. The proper distance from $r=0$ to the cosmic horizon is given by $\ell=\pi L / 2$. The horizon collapses in this limit thus the whole space collapses to a segment of length $\ell$. One may postulate that the system is described by a $1+1$ dimensional CFT. In fact, in the whole physical range of $m$, the subtracted energy and the entropy behave as if there is a CFT

$$
\begin{aligned}
& \triangle M=\frac{\pi L}{G} \ell T_{c}^{2}, \\
& \triangle S_{c}=\frac{2 \pi L}{G} \ell T_{c} .
\end{aligned}
$$

One may be tempted to guess that the whole de Sitter gravity is dual to a $2 \mathrm{~d}$ CFT, this is certainly misleading, since as we said before that a state with a massive particle sitting at $r=0$ is hardly the generic state of the given energy. However, the limit $m=1 /(8 G)$ is special, since the metric collapses to $d s^{2}=d \rho^{2}$, where $\rho=L \sin ^{-1}\left(\frac{r}{r_{c}}\right)$, so one may propose that near this limit there is a $2 \mathrm{~d}$ CFT with central charge $c=6 L / G$ living on the segment.

To summarize, we take the Nariai black hole as the ground state and made one conjecture and two observations on the thermodynamical behavior of Schwarzschild-de Sitter space in three different regions. We conjecture that near the Nariai limit the system is dual to a $2 d$ CFT living on the finite segment stretched between the cosmic horizon and 
the black hole horizon. One observation is that in the pure de Sitter phase, the system's behavior is similar to the Hagedorn string in a fixed volume in $2+1$ spacetime and another one is that the imaginary high temperature phase of quantum gravity in $n+1$ dimensional dS spacetime is dual to a $(n-1)+1$ dimensional CFT. Of course it is a long way to go to reach any certain conclusion about the de Sitter quantum gravity. We hope that the conjecture and observations made here will be useful in the on-going search for the foundation of the de Sitter gravity.

\section{Acknowledgments}

The work of QGH was supported by a grant from NSFC, a grant from China Postdoctoral Science Foundation and and a grant from K. C. Wang Postdoctoral Foundation. The work of KK and ML was supported by a grant from CAS and a grant from NSFC. 


\section{References}

[1] E. Witten, hep-th/0106109.

[2] T. Banks, hep-th/0007146; M. Li, hep-th/0106184, JHEP 0204 (2002) 005; T. Banks, astro-ph/0305037; M. K. Parikh and E. Verlinde, hep-th/0403140; hep-th/0410227, JHEP 0501 (2005) 054.

[3] G. W. Gibbons and S. W. Hawking, Phys. Rev. D 15 (1977) 2738.

[4] Y. Myung, Mod.Phys.Lett. A16 (2001) 2353; Y. Myung, Phys.Lett. B579 (2004) 205210; S. Nojiri and S. Odintsov, Phys.Lett. B523 (2001) 165-170; M. I. Park, Phys. Lett. B440,275 (1998); M. I. Park, Nucl. Phys. B544, 377 (1999).

[5] V. Balasubramanian, Jan de Boer and D. Minic, Phys. Rev. D 65 (2002) 123508, hep-th/0110108.

[6] R. Bousso, JHEP 0011:038, 2000 hep-th/0010252.

[7] R. Bousso and S. W. Hawking, Phys. Rev. D 57 (1998) 2436, hep-th/9709224.

[8] M. Spradlin, A. Strominger and A. Volovich, hep-th/0110007.

[9] A. Strominger, JHEP 0110 (2001) 034, hep-th/0106113; A. Strominger, JHEP 0111 (2001) 049. 